\title{
Harmonic coordinate method for simulating generic singularities
}

\author{
David Garfinkle \\ Department of Physics, Oakland University, Rochester, Michigan 48309
}

\begin{abstract}
This paper presents both a numerical method for general relativity and an application of that method. The method involves the use of harmonic coordinates in a $3+1$ code to evolve the Einstein equations with scalar field matter. In such coordinates, the terms in Einstein's equations with the highest number of derivatives take a form similar to that of the wave equation. The application is an exploration of the generic approach to the singularity for this type of matter. The preliminary results indicate that the dynamics as one approaches the singularity is locally the dynamics of the Kasner spacetimes.
\end{abstract}

04.25.Dm, 04.20.Dw

\section{INTRODUCTION}

It has long been known that harmonic coordinates are useful for mathematical relativity. In particular, these coordinates were used [1] to prove local existence of solutions of the vacuum Einstein equation. This usefulness of harmonic coordinates stems from their putting Einstein's equation into a form that is similar to the curved spacetime wave equation. Since many numerical techniques work well on the wave equation, one might expect that harmonic coordinates would be used extensively in numerical relativity, and it is somewhat surprising that they are not. (However see [2] for some recent mathematical and numerical work on the linearized case. In addition, harmonic time slices have been advocated and used in numerical relativity [3/7). This is perhaps due to the following drawback of harmonic coordinates: these coordinates are solutions of the wave equation, and such solutions need not have a timelike gradient at all points of spacetime, even if they start out with a timelike gradient on an initial data surface. What this means is that in harmonic coordinates, the time coordinate will in general not remain timelike and this is likely to cause numerical problems. [8.9] As we will see later, there is a way around this difficulty.

One area of numerical relativity where harmonic coordinates have been used (though somewhat unintentionally) is in the study of the approach to the singularity, in particular in the Gowdy spacetimes. 10] Numerical simulation of approach to a spacetime singularity presents problems of its own. Since it is expected that various quantities become infinite at the singularity, the numerical simulation will generally stop after a finite coordinate time, the time when a surface of constant time first encounters the singularity. Since this first encounter generally occurs at one spatial point, the information about the behavior of the singularity at other spatial points will be unavailable from the numerical simulation. The solution to this difficulty is to choose a time coordinate that tends to infinity as the singularity is approached. In this way, the simulation is not forced to end at finite coordinate time, the whole spacetime up to the singularity is covered and the behavior of the metric as the singularity is approached simply becomes asymptotic behavior in the limit of large time coordinate. For Gowdy spacetimes there is a natural choice of such a time coordinate: these spacetimes are foliated by $T^{2} \mathrm{~s}$ invariant under the symmetry group. The area of the symmetry $T^{2} \mathrm{~s}$ goes to zero as the singularity is approached. Therefore minus the logarithm of this area is a natural time coordinate that goes to infinity as the singularity is approached. While this method works well for the Gowdy spacetimes, since it depends crucially on the symmetry of the Gowdy spacetimes, the method does not seem to generalize to the case of the generic singularity with no symmetries. A natural generalization comes when one notices that this time coordinate (minus the logarithm of the area of the symmetry $T^{2} \mathrm{~s}$ ) is also harmonic. Since in some sense one expects the wave equation to become singular as the spacetime singularity is approached, one might also expect a solution of the wave equation to blow up as the singularity is approached and thus one might want to use such a solution as the coordinate time.

What is expected to be the generic behavior of a spacetime as the singularity is approached? Based on studies of spacetimes with $T^{2}$ symmetry 10 12] and spacetimes with $U(1)$ symmetry [13], the expected answer [14] is the following: the singularity is expected to be spacelike and as it is approached each spatial point is expected to "decouple" from the others and undergo a dynamics corresponding to that of a homogeneous spacetime (though a different homogeneous spacetime at each spatial point). Which types of homogeneous spacetime is this dynamics expected to correspond to? For vacuum spacetimes, it is thought that the dynamics will be oscillatory, possibly corresponding to the Mixmaster spacetime as conjectured in [15]. For many other types of matter it is expected that as the singularity is approached the matter terms in the Einstein equations become negligible and the dynamics approaches that of a vacuum spacetime.

However for so called "stiff matter" (i.e. a scalar field or a perfect fluid with equation of state $P=\rho$ ) it is ex- 
pected that the dynamics will not be oscillatory and will correspond to that of a Kasner spacetime. This expectation is greatly bolstered by a theorem due to Andersson and Rendall [16] which shows local existence in a neighborhood of the singularity of solutions to the Einsteinscalar equations, with the expected asymptotic behavior and with enough degrees of freedom to be the generic solutions.

Since the approach to the singularity is expected to be simpler for stiff matter than for vacuum, the stiff matter case should be easiser to treat numerically. Therefore, in this paper we confine ourselves to a numerical study of the approach to the generic singularity in the Einsteinscalar system. Section 2 presents the equations and numerical methods used. The results are given in section 3 . Section 4 contains a discussion of the results and of other possible applications of the harmonic coordinate method.

\section{EQUATIONS AND NUMERICAL METHODS}

The equations that we wish to evolve numerically are the Einstein-scalar equations

$$
R_{\alpha \beta}=8 \pi \nabla_{\alpha} \phi \nabla_{\beta} \phi
$$

Here, we use the conventions of [17] including units where $c=G=1$. As a consequence of Eq. (11) and the Bianchi identities, the scalar field must satisfy the wave equation

$$
\nabla_{\alpha} \nabla^{\alpha} \phi=0
$$

The Ricci tensor is given in terms of the Christoffel symbols by

$$
R_{\alpha \beta}=\partial_{\gamma} \Gamma_{\alpha \beta}^{\gamma}-\partial_{\alpha} \Gamma_{\gamma \beta}^{\gamma}+\Gamma_{\alpha \beta}^{\gamma} \Gamma_{\nu \gamma}^{\nu}-\Gamma_{\alpha \nu}^{\gamma} \Gamma_{\beta \gamma}^{\nu}
$$

while the Christoffel symbols are given in terms of the metric by

$$
\Gamma_{\alpha \beta}^{\gamma}=\frac{1}{2} g^{\gamma \delta}\left(\partial_{\alpha} g_{\beta \delta}+\partial_{\beta} g_{\alpha \delta}-\partial_{\delta} g_{\alpha \beta}\right)
$$

Harmonic coordinates are solutions of the wave equation. As a generalization of harmonic coordinates, consider coordinates that satisfy the wave equation with source

$$
\nabla^{\alpha} \nabla_{\alpha} x^{\mu}=H^{\mu}
$$

where $H^{\mu}$ are specified from the beginning. Then using Eqs. (3, 4, 5) we find that the Ricci tensor is given by

$$
\begin{gathered}
R_{\alpha \beta}=-\frac{1}{2} g^{\gamma \sigma} \partial_{\gamma} \partial_{\sigma} g_{\alpha \beta}+\frac{1}{2} C_{\beta}{ }^{\mu \nu} C_{\mu \nu \alpha} \\
+\frac{1}{2} C_{\alpha}{ }^{\mu \nu} C_{\mu \nu \beta}-\Gamma_{\nu \alpha}^{\gamma} \Gamma_{\gamma \beta}^{\nu}-\partial_{(\alpha} H_{\beta)}+\Gamma_{\alpha \beta}^{\gamma} H_{\gamma}
\end{gathered}
$$

where $C_{\alpha \mu \nu} \equiv \partial_{\alpha} g_{\mu \nu}$. Note that the second derivative terms appear only in the wave operator. Therefore, one might expect that Einstein's equations in this form behave similarly to the wave equation and that numerical methods that work well on the wave equation might work well on Einstein's equations in this form. The reason for considering nonzero source terms $H^{\mu}$ in Eq. (5) is that these terms allow us to change the behavior of the time coordinate and thus may allow us to eliminate (or at least postpone) the behavior where the time coordinate ceases to be timelike. In the simulations done in this paper, no source terms were needed. I know of no systematic way to find appropriate source terms and expect that in the cases where they are needed, one will have to resort to a trial and error method to find appropriate $H^{\mu}$. Note that the use of spatial harmonic coordinates could also lead to coordinate problems. This would occur if the gradients of the four coordinates fail to be linearly independent. If this sort of problem occurs, one might expect to be able to postpone or eliminate it by using appropriate source terms.

The numerical method used requires equations that are first order in time. To put the equations in such a form, we define quantities $P_{\alpha \beta}$ and $P_{\phi}$ given by

$$
\begin{gathered}
P_{\alpha \beta}=\partial_{t} g_{\alpha \beta} \\
P_{\phi}=\partial_{t} \phi
\end{gathered}
$$

Then the Einstein-scalar equation becomes

$$
\begin{array}{r}
-g^{00} \partial_{t} P_{\alpha \beta}=2 g^{0 k} \partial_{k} P_{\alpha \beta}+g^{i k} \partial_{i} \partial_{k} g_{\alpha \beta} \\
+16 \pi \partial_{\alpha} \phi \partial_{\beta} \phi+2 \partial_{(\alpha} H_{\beta)}-2 \Gamma_{\alpha \beta}^{\gamma} H_{\gamma} \\
-C_{\alpha}{ }^{\mu \nu} C_{\mu \nu \beta}-C_{\beta}{ }^{\mu \nu} C_{\mu \nu \alpha}+2 \Gamma_{\nu \alpha}^{\gamma} \Gamma_{\gamma \beta}^{\nu}
\end{array}
$$

The wave equation for $\phi$ becomes

$$
-g^{00} \partial_{t} P_{\phi}=2 g^{0 k} \partial_{k} P_{\phi}+g^{i k} \partial_{i} \partial_{k} \phi-g^{\alpha \beta} \Gamma_{\alpha \beta}^{\gamma} \partial_{\gamma} \phi
$$

The full set of equations that are evolved in the computer code are Eqs. (710).

We now turn to the numerical methods used to evolve these equations. Spatial derivatives are approximated by centered differences. The variables are evolved in time using a three step iterated Crank-Nicholson (ICN) method. 18 20 This works as follows: evolution equations of the form $\partial_{t} S=W(S)$ for some set of variables $S$ are approximated as

$$
S^{n+1}=S^{n}+\frac{\Delta t}{2}\left[W\left(S^{n}\right)+W\left(S^{n+1}\right)\right]
$$

where $S^{n}$ is the value of $S$ at time step $n$ and $\Delta t$ is the time step. Then, using $S^{n}$ as an initial guess for $S^{n+1}$, Eq. (11) is iterated three times.

The spacetimes we consider have topology $T^{3} \times R$. Each spatial slice has topology $T^{3}$. In terms of the spatial coordinates, this means that $0 \leq x \leq 2 \pi$ with 0 and $2 \pi$ identified (and correspondingly for $y$ and $z$ ). This topology is implemented numerically as follows: a spatial coordinate $x$ has $N$ grid points. The variables on points from 2 to $N-1$ are evolved using the evolution equations. The variables on point 1 are set to the values at point $N-1$ while at point $N$ they are set to the values at point 2 . 


\section{RESULTS}

All runs were done in double precision on Compaq XP1000 workstations and on the NCSA Origin 2000. The time step was $\Delta t=\Delta x / 2$. While the source terms $H^{\mu}$ should be helpful in keeping the coordinates well behaved, we did not need them for the cases studied here and all runs have $H^{\mu}=0$.

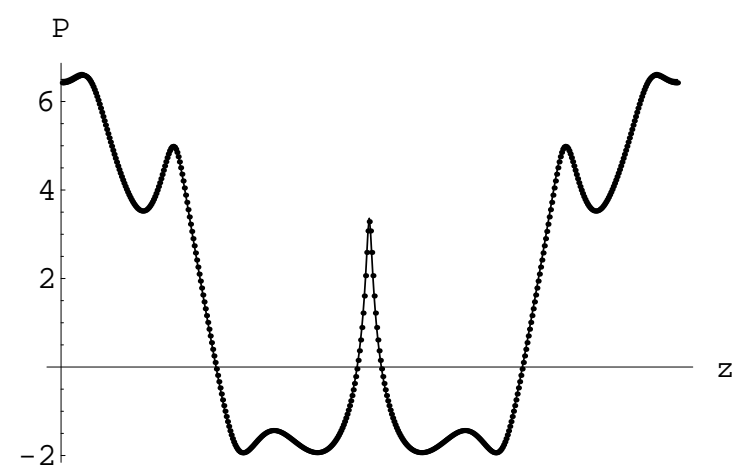

FIG. 1. comparison of the quantity $P$ as found using a Gowdy code and the $3+1$ harmonic code

Before exploring the generic singularity, we would like to test the code. One way of doing this is to run the code on cases with symmetry where results are already known. Such a case is the Gowdy spacetimes. These have the form 10

$$
\begin{array}{r}
d s^{2}=e^{(\tau-\lambda) / 2}\left[-e^{-2 \tau} d \tau^{2}+d z^{2}\right] \\
+e^{-\tau}\left[e^{P} d x^{2}+2 e^{P} Q d x d y+\left(e^{P} Q^{2}+e^{-P}\right) d y^{2}\right]
\end{array}
$$

Here, $P, Q$ and $\lambda$ are functions of $\tau$ and $z$. It follows from Eq. (12) that the coordinates $(\tau, x, y, z)$ are all solutions of the wave equation. Therefore, they are harmonic coordinates. The vacuum Einstein equations for the Gowdy spacetimes are 11]

$$
\begin{gathered}
\partial_{\tau} \partial_{\tau} P-e^{-2 \tau} \partial_{z} \partial_{z} P-e^{2 P}\left[\left(\partial_{\tau} Q\right)^{2}-e^{-2 \tau}\left(\partial_{z} Q\right)^{2}\right]=0 \\
\partial_{\tau} \partial_{\tau} Q-e^{-2 \tau} \partial_{z} \partial_{z} Q+2\left(\partial_{\tau} P \partial_{\tau} Q-e^{-2 \tau} \partial_{z} P \partial_{z} Q\right)=0
\end{gathered}
$$

plus constraint equations that determine $\lambda$ once $P$ and $Q$ are known.

To test the $3+1$ code, we evolve Eqs. (13) and (14) using a $1+1$ code. Then we evolve the same initial data with the $3+1$ harmonic code and compare the results. To do the comparison, note that $P=\tau+\ln g_{x x}$ so that it is straightforward to compare the values of $P$ produced by the two codes. For these simulations 500 gridpoints were used in the $1+1$ code. In the $3+1$ code, 3 gridpoints were used in the $x$ direction, 3 gridpoints in the $y$ direction and 500 gridpoints in the $z$ direction. For the comparison the initial data used is $P=0, \partial_{\tau} P=5 \cos z, Q=\cos z, \partial_{\tau} Q=0$. These data are evolved until $\tau=\pi$ and the results for the comparison are given in Fig. 1. Here, the solid line represents the $1+1$ evolution of the Gowdy equations, while the dots represent the full $3+1$ evolution using the harmonic code. There is clearly agreement between the two.

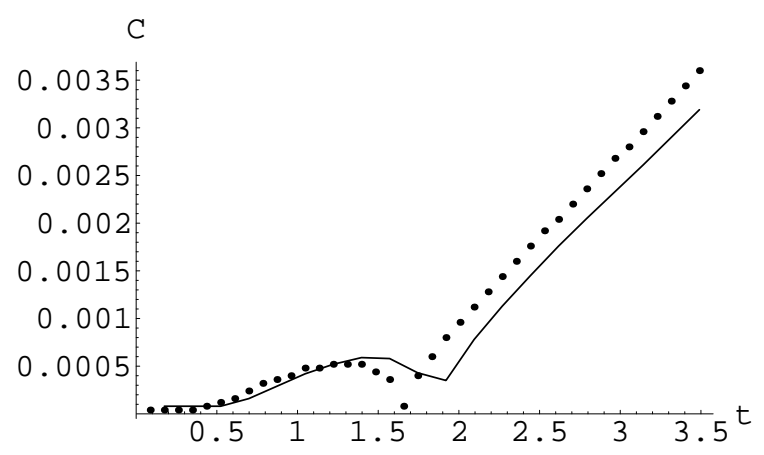

FIG. 2. convergence test involving the constraint

Before presenting the results of another code test, we turn to the question of finding initial data without symmetries. On an initial data surface, the intrinsic metric $h_{i j}$ and the extrinsic curvature $K_{i j}$ must satisfy the constraint equations

$$
\begin{gathered}
D_{i} K^{i}{ }_{j}-D_{j} K=8 \pi \dot{\phi} D_{j} \phi \\
{ }^{(3)} R+K^{2}-K^{i j} K_{i j}=8 \pi\left[\dot{\phi}^{2}+D^{i} \phi D_{i} \phi\right]
\end{gathered}
$$

(Note that we use the convention of [17] for the sign of $K_{i j}$ which is opposite to the convention usually used in numerical relativity). Here, an overdot denotes derivative along the normal to the surface, and $D_{i}$ and ${ }^{(3)} R$ are respectively the covariant derivative and scalar curvature of $h_{i j}$. Given a solution of Eqs. (15) and (16), we produce initial data for evolution in harmonic coordinates as follows: For spatial directions $i$ and $j$ we have $g_{i j}=h_{i j}, g_{i 0}=g_{0 i}=0, g_{00}=-1, P_{i j}=2 K_{i j}$. The remaining components of $P_{\alpha \beta}$ are solved for using Eq. (5).

We want to find a solution of Eqs. (15) and (16) that is simple but has no symmetries and has some free parameters. We choose $\phi=0$ and $h_{i j}$ equal to the flat Euclidean metric in the usual coordinates. For the extrinsic curvature we choose

$$
\begin{array}{r}
K_{x x}=\left(b_{1}+a_{2} \cos y+a_{3} \cos z\right) / 2 \\
K_{y y}=\left(b_{2}+a_{1} \cos x-a_{3} \cos z\right) / 2 \\
K_{z z}=\left(b_{3}-a_{1} \cos x-a_{2} \cos y\right) / 2 \\
K_{x y}=K_{y x}=\left(c_{1} \cos z\right) / 2 \\
K_{x z}=K_{z x}=\left(c_{2} \cos y\right) / 2
\end{array}
$$




$$
K_{y z}=K_{z y}=\left(c_{3} \cos x\right) / 2
$$

Here, the quantities $a_{i}, b_{i}$ and $c_{i}$ are constants that are free parameters. It is straightforward to show that the extrinsic curvature of Eq. (17) with our choice of initial $h_{i j}$ and $\phi$ satisfies Eq. (15). Equation (16) then becomes an agebraic equation for $P_{\phi}$ which can be solved provided that the left hand side of the equation is positive. Note that if all the $a_{i}$ and $c_{i}$ are zero and all the $b_{i}$ are equal, then the initial data evolve to the spatially flat Robertson-Walker spacetime with scalar field matter. Thus, this family of data can be thought of as RobertsonWalker with large gravitational and scalar waves.

We now consider a convergence test involving a constraint that comes from the use of harmonic coordinates. Define the quantities $C^{\mu}$ by

$$
C^{\mu}=g^{\alpha \beta} \Gamma_{\alpha \beta}^{\mu}
$$

(this is the appropriate form of the constraint for the case where the source term $H^{\mu}$ vanishes. For the general case, the constraint would be given by $C^{\mu}=g^{\alpha \beta} \Gamma_{\alpha \beta}^{\mu}+$ $H^{\mu}$ ). Then from Eq. (5) it follows that $C^{\mu}=0$. Since we are solving the evolution equations by approximating them by finite difference equations, the quantities $C^{\mu}$ as evaluated by the computer code will not be zero because of errors due to the finite grid spacing $\Delta x$. Define the quantity $C$ by

$$
C=\left|\frac{\int \sqrt{g} g_{\alpha \beta} C^{\alpha} C^{\beta} d x d y d z}{\int \sqrt{g} d x d y d z}\right|^{1 / 2}
$$

This quantity is a type of measure of the average size of the constraint. Figure 2 shows a plot of $C$ vs. time. The parameters used are $a_{i}=(0.1,0.1,0.2), b_{i}=$ $(0,-0.5,-0.5)$ and $c_{i}=(0,0,0)$. Here the curve corresponds to a run done with 20 gridpoints in each spatial direction, while the dots correspond to a run with 38 gridpoints in each spatial direction (which gives half the grid spacing) and with $C$ multiplied by 4 . The results show second order convergence.

We now consider the approach to the singularity. To see what is expected, it is helpful to consider the Kasner spacetime in harmonic coordinates. This is given by

$$
d s^{2}=-e^{\left(q_{1}+q_{2}+q_{3}\right) \tau} d \tau^{2}+e^{q_{1} \tau} d x^{2}+e^{q_{2} \tau} d y^{2}+e^{q_{3} \tau} d z^{2}
$$

where the $q_{i}$ are constants This metric is generally a solution of the Einstein-scalar equations; but for the case where $\left(\sum q_{i}\right)^{2}=\sum q_{i}^{2}$ it is a vacuum spacetime. (In the usual treatment of vacuum Kasner spacetimes, one defines the quantities $p_{i}=q_{i} /\left(\sum q_{m}\right)$ and then obtains the condition $\left.\sum p_{i}=\sum p_{i}^{2}=1\right)$. Note that in the vacuum case one cannot have all three directions contracting but that this is possible for the Einstein-scalar case. Note also that the metric components are exponential functions of time. It also turns out that the scalar field

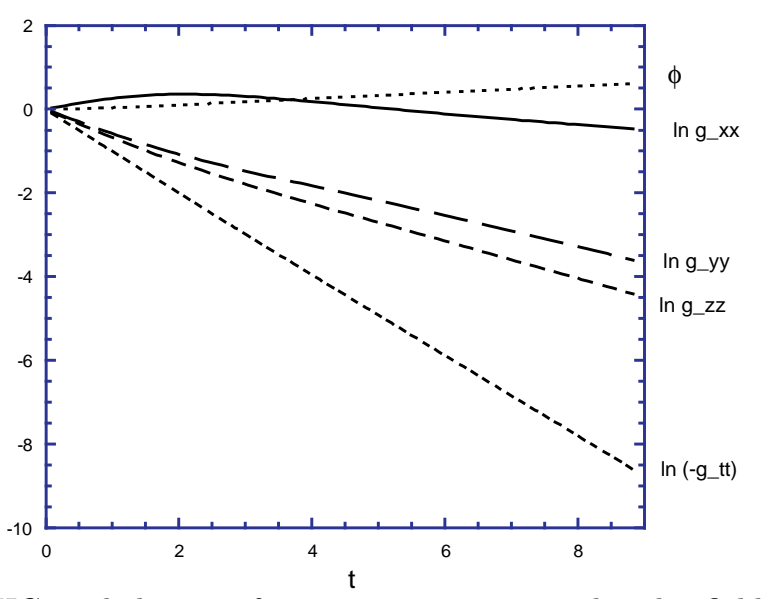

FIG. 3. behavior of metric components and scalar field as the singularity is approached at the spatial point $(0,0,0)$

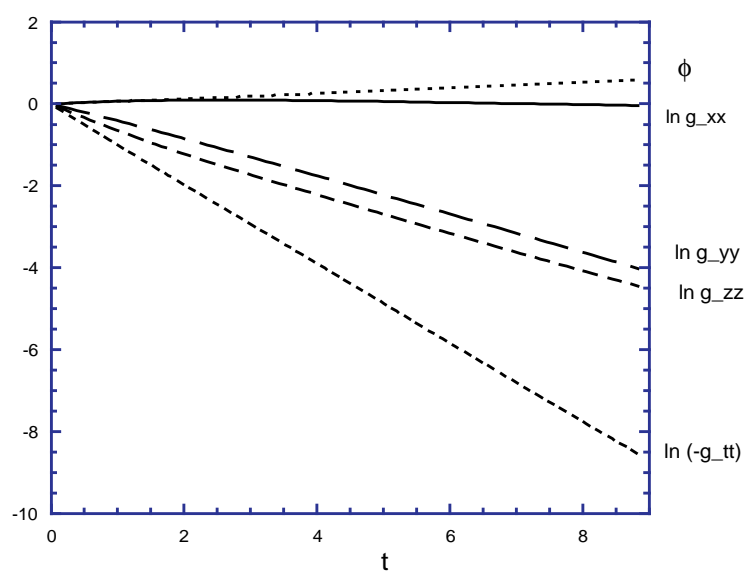

FIG. 4. behavior of metric components and scalar field as the singularity is approached at the spatial point $(0, \pi / 4, \pi / 2)$

is a linear function of time. Thus, if the behavior of a generic solution near the singularity is local Kasner, then we should expect metric components that are exponential functions of time, with the exponent depending on space. We should also expect a scalar field that is a linear function of time with the slope depending on space.

Results on the approach to the singularity are shown in Figs. 3 and 4. The run was done with 34 grid points in each spatial directions. The parameters $a_{i}, b_{i}$ and $c_{i}$ are the same as for the convergence test. Here the scalar field and the logarithms of the diagonal metric components are plotted as functions of time. Figure 3 corresponds to the spatial point $(x, y, z)=(0,0,0)$ while for Fig. 4 the point is $(0, \pi / 4, \pi / 2)$. Note that as the singularity is approached these quantities all become linear functions of time. The differences between Figs. 3 and 4 show that there is a spatial dependence of the approach to the singularity. Note from Fig. 3 that though the $x$ direction is initially expanding, eventually all three directions 
contract. This is what one would expect if the metrics of [16] represent the generic behavior near the singularity since these metrics have all three directions contracting in a neighborhood of the singularity.

\section{DISCUSSION}

While this study is somewhat preliminary, it indicates that harmonic coordinates can be a useful tool in numerical relativity. Though, in principle coordinate problems could occur, this did not happen in the cases studied here, even though they involved very strong fields. Furthermore, the use of the source terms $H^{\mu}$ may cure such problems if they arise.

As for the behavior of generic singularities, the numerical results indicate that solutions of the form proved in reference [16] to exist in a neighborhood of the singularity also exist globally. Thus such solutions are likely to describe the generic singularity in the stiff matter case.

There are several projects for which the methods of this paper could be used. One is to do a more extensive study of the singularity in the Einstein-scalar case, with a more thorough exploration of the evolution corresponding to various values of the parameters in the initial data of the previous section. It would also be helpful to evolve for a longer time.

Another project is to remove the scalar field and study the approach to the singularity of the generic vacuum spacetime. Here, the behavior is expected to be more complicated and a treatment will probably require more spatial resolution to resolve the expected sharp features, as well as longer evolution in time to see the expected oscillatory behavior.

Yet another project is to study the behavior of asymptotically flat spacetimes rather than closed cosmologies. Here, the closed cosmologies were studied partly for simplicity. The periodic boundary conditions are simple to implement and completely consistent with Einstein's equations. In contrast, for an outer boundary at a finite distance in an asymptotically flat spacetime, one needs to put some sort of outgoing wave boundary condition. Such conditions are usually not consistent with Einstein's equation (it is known how to have a consistent boundary condition 21 but this condition is quite complicated). These inconsistent conditions may lead to numerical instability. Since harmonic coordinates make Einstein's equation look like the wave equation, simple outgoing wave boundary conditions that work numerically with the wave equation might be expected to "work" (at least in the sense of not causing numerical instability) for Einstein's equation.

Since much work has been done on numerical simulations of asymptotically flat spacetimes using the standard Arnowitt-Deser-Misner (ADM) 22 approach, it is helpful to make comparisons with this approach. In the ADM approach, the spacetime metric is written as

$$
d s^{2}=-\alpha^{2} d t^{2}+h_{i j}\left(d x^{i}+\beta^{i} d t\right)\left(d x^{j}+\beta^{j} d t\right)
$$

Einstein's equations are written as an evolution equation for the spatial metric $h_{i j}$ (and the extrinsic curvature $K_{i j}$ ) while the gauge choice results in equations for the lapse $\alpha$ and the shift $\beta^{i}$. This framework is sufficiently general to accomodate the use of harmonic coordinates, which correspond to the following equations for lapse and shift

$$
\begin{gathered}
\partial_{t} \alpha-\beta^{i} \partial_{i} \alpha=K \alpha^{2} \\
\partial_{t} \beta^{i}-\beta^{m} \partial_{m} \beta^{i}=h^{m n} \bar{\Gamma}_{m n}^{i} \alpha^{2}
\end{gathered}
$$

(Note that the sign of $K$ in equation (22) comes from the convention of $[17]$ ). Here $\bar{\Gamma}_{m n}^{i}$ is the Christoffel symbol associated with $h_{i j}$. (Equations (22) and (23) hold for the case of vanishing source term $H^{\mu}$. Similar equations hold in the case of nonzero $H^{\mu}$ ). The use of equations (22) and (23) in an ADM code is not precisely equivalent to the approach of this paper. The reason for this is that equations (22) and (23) directly solve the constraint $g^{\alpha \beta} \Gamma_{\alpha \beta}^{\mu}=0$, while in our approach, this constraint is used to change the form of the evolution equations. Nonetheless, it would be interesting to use equations (22) and (23) in an ADM code to see how that compares with other choices of lapse and shift. In particular, one might expect better stability properties and compatibility with a simple outgoing wave boundary condition. (Though note that such improvements can also be obtained using the BSSN approach [23 [25]).

Another desired feature of a numerical code is the ability to treat black holes, and this sometimes requires black hole excision. While harmonic coordinates are singularity avoiding [3] they are just barely so and come arbitrarily close to a singularity. Thus the need for excision in an approach that uses harmonic coordinates should be at least as great as in the standard approach. Nonetheless, one might hope that excision itself would be easier to implement using the approach of this paper. This is because no elliptic equations are involved and the light cone of the wave operator is the same as that of the physical metric.

All these projects are work in progress and preliminary results from them are promising. Thus, I expect that harmonic coordinates will become a useful tool in numerical relativity.

\section{ACKNOWLEDGEMENTS}

I would like to thank Helmut Friedrich, Beverly Berger, Matt Choptuik, G. Comer Duncan, Lars Andersson and Alan Rendall for helpful discussions. I would also like to thank the Albert Einstein Institute for hospitality. This work was partially supported by NSF grant PHY9988790 to Oakland University. Some of the computations were performed at the National Center for Supercomputing Applications (Illinois). 
[1] Y. Foures-Bruhat, Acta Mathematica, 88, 141 (1952)

[2] B. Szilagyi, B. Schmidt and J. Winicour, gr-qc/0106026

[3] C. Bona and J. Masso, Phys. Rev. D38, 2419 (1988)

[4] C. Bona and J. Masso, Phys. Rev. D40, 1022 (1989)

[5] C. Bona and J. Masso, Phys. Rev. D68, 1097 (1992)

[6] A. Abrahams, A. Anderson, Y. Choquet-Bruhat and J. York, Phys. Rev. Lett. 75, 3337 (1995)

[7] P. Anninos, K. Camarda, J. Masso, E. Seidel, W. Suen and J. Towns, Phys. Rev. D52, 2059 (1995)

[8] M. Alcubierre, Phys. Rev. D55, 5981 (1997)

[9] M. Alcubierre and J. Masso, Phys. Rev. D57, 4511 (1998)

[10] B. Berger and V. Moncrief, Phys. Rev. D48, 4676 (1993)

[11] B. Berger and D. Garfinkle, Phys. Rev. D57, 4767 (1998)

[12] B. Berger, J. Isenberg and M. Weaver, Phys. Rev. D64, 084006 (2001)

[13] B. Berger and V. Moncrief, Phys. Rev. D58, 064023 (1998)

[14] B. Berger, D. Garfinkle, J. Isenberg, V. Moncrief and M. Weaver, Mod. Phys. Lett. A13, 1565 (1998)

[15] V. Belinskii, I. Khalatnikov and E. Lifshitz, Sov. Phys. Usp. 13, 745 (1971)

[16] L. Andersson and A. Rendall, Commun. Math. Phys. 218, 479 (2001)

[17] R.M. Wald, General Relativity (University of Chicago Press, Chicago, 1984)

[18] M. Choptuik, in Deterministic Chaos in General Relativity D. Hobill, A. Burd and A. Coley eds. (Plenum, New York, 1994) pp. 155-175

[19] S. Teukolsky, Phys. Rev. D61, 087501 (2000)

[20] M. Alcubierre et al, Phys. Rev. D62, 044034 (2000)

[21] H. Friedrich and G. Nagy: Commun. Math. Phys. 201, $619(1999)$

[22] R. Arnowitt, S. Deser and C. Misner, in Gravitation: an introduction to Current Research, L. Witten ed. (John Wiley, New York, 1962) pp. 254-280

[23] T. Nakamura, K. Oohara and Y. Kojima, Prog. Theor. Phys. Suppl. 90, 1 (1987)

[24] M. Shibata and T. Nakamura, Phys. Rev. D52, 5428 (1995)

[25] T. Baumgarte and S. Shapiro, Phys. Rev. D59, 024007 (1999) 